\title{
Progress and Problems with Viral Vectors for Delivery of TALENS
}

\section{Thorsten Bergmann, Eric Schulz and Anja Ehrhardt*}

Institute of Virology and Microbiology, Center for Biomedical Education and Research, Department of Human Medicine, Faculty of Health, University Witten/Herdecke, Germany

\begin{abstract}
It has long been envisaged that gene disruption or gene correction in affected target cells can be efficiently conducted in vitro and in vivo and over the recent years several tools for achieving this goal were developed. Designer nucleases such as zinc finger nucleases (ZFNs) were extensively explored and more recently transcription activator-like effector nucleases (TALENs) were introduced for sequence-specific genome engineering in the mammalian genome. ZFNs and TALENs are fusion proteins containing a customized DNA-binding motif for sequence-specific DNA binding linked to a nuclease for introduction of double-stranded DNA breaks. Both systems were explored in mammalian cells using non-viral and viral delivery methods. Herein, we will provide a state-ofthe-art overview of available virus-based delivery systems for sufficient expression of functional TALENs. We will cover the molecular design of recombinant viruses containing TALEN expression cassettes and we will mention advantages and disadvantages of the respective systems. Although the relevance of these viral vector systems for novel developments in molecular medicine and genome engineering need to be further evaluated, we believe that with further improvements these viral vectors for TALEN delivery will play an emerging role in bioengineering and for establishing novel therapeutic concepts.
\end{abstract}

Keywords: TALEN; Viral vector, Delivery; Adenovirus; Lentivirus; Baculovirus; Gene disruption; Gene editing

\section{Transcription Activator-Like Effector Nucleases (TALENS) and Their Mode of Action}

For sequence-specific genome engineering and its biotechnological and medical applications various systems were tested. Most recent tools include designer nucleases and the bacteria derived clustered regularly interspaced short palindromic repeats (CRISPR)/Cas9 system using short RNA to induce precise cleavage at endogenous genomic loci $[1,2]$. Designer nucleases are mainly represented by zinc finger nucleases (ZFNs) and transcription activator-like effector nucleases (TALENs) [3-7], combining features of a customized DNA binding motif for sequence-specific DNA binding domain and a nuclease for introduction of doubled-strand DNA (dsDNA) breaks at the target site.

TALENs are a promising new class of designer nucleases that can be specifically designed to bind DNA sequences of interest and to introduce dsDNA breaks. They are chimeric proteins consisting of a N-terminal nuclear localization signal, a central DNA binding domain and a C-terminal FokI nuclease domain. The DNA binding domain originates from transcription activator like effectors (TALEs) of the bacterial plant pathogen Xanthomonas, used by this bacterium to alter the expression of several host genes [8]. Its main characteristic is a central repeat region consisting of a variable number of incomplete tandem repeats that are usually comprised of 33-35 amino acids (aa) that are identical except for the hypervariable repeat-variable diresidue (RVD) at a positions 12 and 13 [6]. With some degree of degeneracy, the $\mathrm{RVD}$ of each repeat is specific for binding a corresponding nucleotide in their contiguous target DNA sequence $[6,8]$. The C-terminal FokI domain provides non-specific endonuclease activity and similar to ZFNs, binding of the TALE domains upstream and downstream of the respective DNA target and subsequent dimerization at the FokI nuclease domain lead to dsDNA breaks. It is of note that the spacer between the TALEN DNA binding sites needs to be considered when designing novel TALENs [6,7]. After binding, dsDNA breaks are introduced which can than activate cellular pathways either leading to homologous recombination in the presence of the respective homologous donor
DNA or stimulation of DNA repair via non homologous end joining (NHEJ). NHEJ usually leads to DNA insertions or deletions causing a specific knockout of respective genes. Therefore, TALENs are valuable tools for genetic engineering as well as cell- and gene therapeutic applications.

Over the recent years several methods arose to specifically design $[6,9]$ and to assemble TALENs and TALE pairs for the desired application in an inexpensive, simple and fast manner [6,10-12]. Assembly can be performed without complex screening procedures or the need for special equipment, making TALEN assembly in this regard superior to the ZFN technology. The potential of TALENs was extensively explored in vitro in eukaryotic cell culture models using co-transfection of TALEN-encoding plasmids $[13,14]$, in vivo using microinjection $[15,16]$, and for detection of TALEN mediated gene disruption or gene correction [17-20].

ZFNs and TALENs are suitable for genome engineering in vitro and in vivo. However, for instance for the majority of in vivo applications and approaches in primary cells, viral vectors may be more efficient for delivery of the respective designer nucleases. For efficient delivery of the ZFNs technology into the desired target cell, commonly used viral vectors including adenoviruses (AdV), lentiviruses (LV) and adenoassociated viruses (AAV) were broadly explored [4,21,22]. However, a limited number of attempts exist for viral delivery of TALENs. The following chapter provides an overview of viral vector types which

*Corresponding author: Anja Ehrhardt, Ph.D., Institute of Virology and Microbiology, Center for Biomedical Education and Research, Department of Human Medicine, Faculty of Health, University Witten/Herdecke, 58453 Witten, Germany, Tel: +49 2302926 273; Fax: + 492302926 44278; E-mail: anja.ehrhardt@uni-wh.de

Received November 11, 2013; Accepted Janyary 13, 2014; Published Janyary 20, 2014

Citation: Bergmann T, Schulz E, Ehrhardt A (2014) Progress and Problems with Viral Vectors for Delivery of Talens. J Mol Genet Med 7: 096 doi: 10.4172/17470862.1000096

Copyright: (c) 2014 Bergmann T, et al. This is an open-access article distributed under the terms of the Creative Commons Attribution License, which permits unrestricted use, distribution, and reproduction in any medium, provided the original author and source are credited 
were studied for TALEN delivery.

\section{Viral Vectors for TALEN Delivery}

Several viral vectors are available for delivery of TALEN expression cassettes which usually consist of the RNA polymerase II (Pol II) promoter, the TALE encoding sequence with an approximate length of 15 to 33 repeats, the nuclease domain and the polyadenylation signal. Here we introduce viral vector systems which were evaluated for delivery of TALEN expression cassettes including vectors based on adenovirus, lentivirus and baculovirus (BV).

AdVs belong to the family of Adenoviridae which are nonenveloped viruses with an icosahedral capsid. The capsid contains the double-stranded DNA genome with a length of 26 to 45 kilo base pairs (kb) dependent on the serotype. There are more than 60 identified human adenoviral serotypes displaying varying tropisms for different cell lines and organs. Historically, the most commonly used adenoviral serotype is the human adenovirus serotype 5 which shows a broad tropism in vitro and in vivo. Wild type adenovirus serotype 5 contains a $36 \mathrm{~kb}$ genome and for generation of first generation AdVs the early gene E1 $(3.15 \mathrm{~kb})$ and the early gene E3 $(3.1 \mathrm{~kb})$ can be deleted. Since the total packaging capacity of an adenoviral genome should not exceed $105 \%$, up to $8.25 \mathrm{~kb}$ of foreign DNA can be encapsidated into the icosahedral capsid. Second generation AdVs lack more than two early genes including E2 and E4, providing an extended genome packaging capacity compared to first generation AdVs [23,24]. However, due to leaky expression of adenoviral genes preserved in the recombinant AdV genome, first and second generation AdVs are not considered as optimal vectors for therapeutic approaches, because de novo production of adenoviral proteins causes enhanced adaptive immune responses including a cytotoxic T-cell response [25]. The most advanced version of recombinant AdVs are represented by high-capacity AdVs (HCAdVs) lacking all viral coding sequences $[26,27]$. They can be distinguished from the other recombinant AdVs by a significantly reduced induction of innate and adaptive immune responses [28]. Only the left and right inverted terminal repeats (ITR) and the packaging signal at the 5' end of the genome are maintained, providing a total packaging capacity of up to $36 \mathrm{~kb}$. It is of note that recombinant AdVs show low integration efficiencies in vitro and in vivo and that they predominantly persist as extrachromosomal DNA vector genomes [29].

LVs are part of the family of Retroviridae and belong to the group of enveloped and single-stranded RNA viruses. LV vectors are derived from the human immune deficiency virus (HIV) and the prototype of these vectors was already developed in 1996 [30]. Early generation LV genomes contain the gene of interest and essential cis-acting elements such as the long terminal repeats (LTRs), the packaging signal and the Rev-responsive element (RRE). To further improve the LV vector system, second generation vectors deleted for multiple non-essential accessory genes were generated [31] and self-inactivating (SIN) vectors were established [32], avoiding unwanted generation of replicationcompetent LVs and the activation of nearby genes due to genomic integration. For third generation LVs "transactivator of transcription" (tat)-independent vectors were generated. Moreover, the central polypurine tract (cPPT) for increased vector transduction efficiency and the woodchuck hepatitis virus post-transcriptional regulatory element (WPRE) for enhanced transgene expression levels were inserted into the recombinant LV genome. Numerous preclinical and clinical trials using LV were performed or are currently being conducted. For achieving stable transgene expression, LVs which have a packaging capacity of up to $8 \mathrm{~kb}$ integrate into the host genome mediated by the HIV-derived integrase. Since LV predominantly integrates into transcriptional active regions in the host genome $[4,33,34]$ causing potentially genotoxic side effects [35], non-integrating lentiviral vectors (IDLVs) were developed [36] carrying a mutation in the catalytic domain of the integrase protein. During infection, genomes of IDLVs form DNA intermediates such as 1- and 2-LTR circles within a cell and it was shown that these LTR circles mediate short-term transgene expression after transduction [37].

BVs belong to the family of Baculoviridae and exhibit a doublestranded circular DNA genome in a filamentous shape. They primarily infect insects but BV derived vectors are also capable of infecting various mammalian cell types including also human embryonic stem cells [38] and pluripotent stem cells $[39,40]$. BV vectors are derived from the well characterized Autographa californica multiple nucleopolyhedrovirus (AcMNPV), which contains a genome of $134 \mathrm{~kb}$ with 155 genes. Since viral proteins polyhedrin and $\mathrm{p} 10$ are dispensable for virus replication, their respective genes can be replaced by transgene expression cassettes. This leads to a total transgene capacity of approximately $38 \mathrm{~kb}$. After transduction, AcMNPV neither replicates nor integrates into the genome of mammalian cells, making it in combination with its simple production and no pre-existing immunity to an efficient vector system for various applications. AcMNVPare extensively used for protein production [41,42], virus production [43,44], vaccine development $[32,45]$ vaccine production [46] and cancer therapy [47]. However, although BV is replication-deficient in mammalian cells, several viral genes are expressed and lead to robust immune responses in vivo $[48,49]$. This effect is reduced in stem cells and therefore, BV derived vectors are primarily useful in regenerative medicine. Several improvements of this vector system were achieved by altering the viral surface protein gp64 with different signal peptides for transduction of certain mammalian cells [50]. Other improvements could be achieved by protecting BV vectors from inhibition factors within serum which lead to more efficient transduction in vitro and in vivo [51].

\section{Molecular Design and Functionality of TALEN Delivering Viral Vectors}

To date there are two studies demonstrating that viral vectors can be explored for delivery of TALEN expression cassettes into target cells $[40,52]$. In the first study lentiviral and adenoviral vectors and their ability to result in sufficient expression of functional TALENs in the desired target cells was investigated. For this purpose a TALEN expression cassette under control of a constitutive cytomegalovirus (CMV) promoter was integrated into different adenoviral and lentiviral vectors. The TALE encoding sequence contained 18 repeats for targeting of the AAVS1 locus within the human genome which represents the predominantly used site of insertion of wild type AAV on chromosome 19 [53]. For the LV constructs third generation vectors including WPRE and CPPT were used whereas for the AdV based approach first and second generation AdVs were employed, either lacking only the early gene E1 or E1 and the early gene region E2A.

For each TALEN expression cassette a single AdV or LV vector was constructed and therefore, simultaneous infection of one cell with two vectors for expression of the functional TALEN pair was required. The strategy of these approaches based on AdV and LV is schematically shown in Figure 1. Different cell types including HeLa cells, immortalized cells from patient affected by Duchenne muscular dystrophy (DMD) and human mesenchymal stem cells (hMSCs, primary cells) were infected. Obtained results demonstrated that the adenoviral constructs of both generations were able to deliver the 
complete TALEN expression cassette into all cell types. TALEN proteins could be detected via western blot analysis and immunofluorescence microscopy. The functionality of the TALEN pair was checked via Surveyor nuclease mutation detection assay for which amplicons of the target locus were denaturated and subsequently re-annealed. In case of mutated sequences this leads to DNA mismatches caused by NHEJ of TALEN-induced dsDNA breaks, which can be detected and cleaved by mismatch-sensitive enzymes. Holkers and colleagues [52] could demonstrate that for AdV-based delivery this cleavage increased in a vector dose-dependent manner. To confirm this result, 20 randomly selected clones were sequenced of which 12 clones were mutated. Overall these findings prove that first and second generation AdVs are valuable tools for viral delivery of complete and functional TALEN expression cassette into human cells.

In case of LV-based delivery of TALEN expression cassettes, it could be shown that both integration competent (ICLV) and integration deficient (IDLV) LVs failed to deliver complete TALEN expression cassettes into target cells [52]. PCR results showed reduced numbers of repeat domains for the integrated proviruses and IDLVs after infection. Subsequent sequencing confirmed that deletion of complete repeats occurred during vector production ranging from 5-15 repeats per TALEN [52]. However, there is evidence that reverse transcriptase (RT) of simple and complex retroviruses possesses low affinity to their RNA template and therefore switching events within the template during the reverse transcription reaction from RNA to DNA may occur [5456]. Due to the fact that the presence of direct repeats increases these switching events resulting in deletion of one or more repeats [54-57], it seems that the usage of commonly used versions of LV vector systems for sufficient delivery and expression of TALENs in mammalian cells is not feasible.

In the second study a BV vector system was used to deliver TALENs into U87 glioblastoma-derived cells and human-induced pluripotent stem cells (h-iPSCs) [40]. Unlike the study by Holkers and colleagues [58], this study was based on a single vector containing both TALEN expression cassettes in one virus genome (BV-TALEN) (Figure 2). It is of note that for the BV system TALENs were tested targeting a different sequence within the AAV-S1 locus when compared to the study exploring AdV and LV as vehicles for TALEN delivery. Nevertheless, TALENs utilized in the BV-based study were confirmed as highly specific for the respective genomic target site via cleavage mismatch-sensitive assay. It could also be demonstrated that transduction of BV-TALEN at moderate MOI resulted in no genomic instability or increased cell death in glioma cells. After co-transduction of U87 cells with BV-TALEN and a $\mathrm{BV}$ vector containing an neomycin-eGFP-expression cassette (Neo-eGFP) flanked by homologous sequences to the AAVS1 locus, Zhu et al. [40] could show stable integration of the Neo-eGFP cassette into the AAVS1 locus. Efficiency was determined by measuringe GFP expression levels, a PCR approach detecting the modified AAVS1 locus, and Southern blot analysis, reaching a value of up to $95 \%$ eGFP positive cells. These results show that high efficiency and specificity of TALENmediated homologous recombination via a viral vector delivery system can be achieved. In order to investigate whether also stem cells could

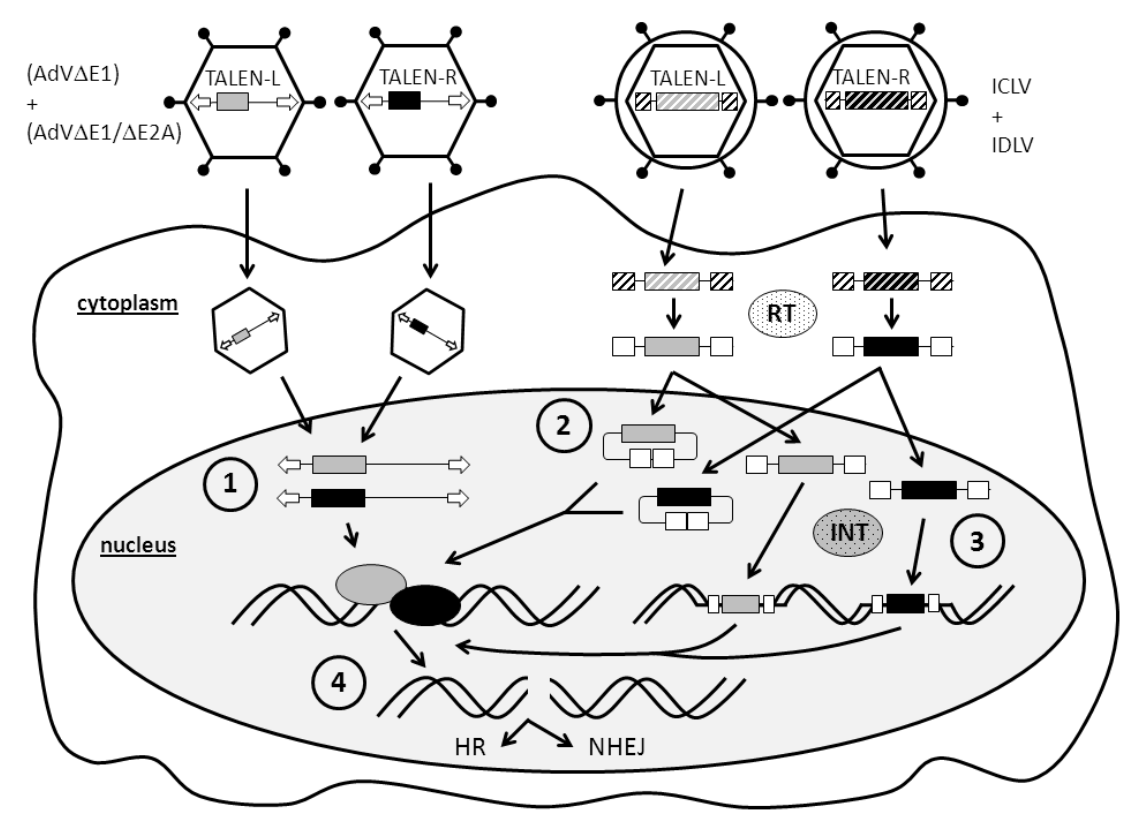

Figure 1: Schematic overview of adenoviral and lentiviral vector systems available for TALEN delivery into mammalian cells. First generation (AdV $\triangle \mathrm{E} 1$ ) and second generation $(A d V \triangle E 1 / \triangle E 2 A)$ adenoviral vectors can be used for delivery of TALEN pairs (TALEN-L and TALEN-R). After nuclear entry adenoviral vectors form linear DNA molecules from which direct mRNA transcription of TALENs occurs (1). Lentiviral vectors (LV) contain RNA based genomes and after entry into the cytoplasm these are transcribed into cDNA by a virus encoded reverse transcriptase (RT). For integration deficient LV (IDLV) vector genomes can exist as episomal 1-LTR and 2-LTR circles from which TALENs can be expressed (2). For integration competent LV vectors (ICLV) integration of cDNA into the host genome is mediated by a virus encoded integrase (INT) (3). Subsequently, expression of TALENs from stable integrated cDNA (provirus) can occur (3). For both vector systems TALEN proteins can bind to the desired DNA target site and TALEN induced double-strand breaks then either lead to non-homologous end joining (NHEJ) or homologous recombination (HR) in the presence of donor DNA with homologous DNA sequences (4). TALEN-L: left part of the TALEN pair; TALEN-R: right part of the TALEN pair; grey horizontal boxes: TALEN-L expression cassette; blackhorizontal boxes: TALEN-R expression cassette; grey oval: TALEN-L protein; black oval: TALEN-R protein; dashed boxes: RNA genomes of lentiviruses encoding TALEN-L and TALEN-R; white horizontal arrows: adenoviral inverted terminal repeats (ITR); white boxes: lentiviral long terminal repeats (LTR); dotted ovals: lentiviral retroviral transcriptase (RT) and lentiviral integrase (INT); AdV $\triangle E 1$ : first generation adenoviral vector deleted for the early gene E1; second generation adenoviral vector deleted for the early genes E1 and E3. 
be site-specifically modified, co-transduction of BV-TALEN and the BV vector with Neo-eGFP was performed in h-iPSCs. After selection with neomycin eGFPexpression could be detected in up to $99.3 \%$ of all selected cells 65 days post-transfection. Site-specific integration of the Neo-eGFP cassette was confirmed via PCR. In summary, these data revealed that TALEN-mediated engineering of stem cells via a BVbased vector system is highly efficient and could play an emerging role in future stem cell based applications.

\section{Risks and Improvements}

Single TALEN expression vectors as performed in the study by Holkers and colleagues [58] require co-infection of two vectors encoding for each part of the nuclease pair. These approaches for adequate TALEN expression especially in vivo are confronted with several problems. First of all both vectors have to infect the same cell with nearly equal efficiency to provide the same amount of each TALEN protein within the cell. Furthermore, a system which relies on co-infection with two viral vectors may require an increased viral dose which could stimulate enhanced toxic side effects and an enhanced immune response. The next challenge could be associated with high expression levels and long-term expression of designer nucleases when using viral vectors for TALEN delivery. For instance the risk of genotoxicity due to off-target effects and therefore activity of TALEN pairs at unwanted loci due to unspecific binding should be analyzed in further detail. Furthermore, activity of the TALEN pair at the targeted chromosomal location in combination with unspecific binding and activity of the same TALEN pair at off-target sites may also lead to chromosomal translocations by linking the two translocation breakpoints. Although there is no direct evidence that chromosomal translocations can be induced after introducing a single TALEN pair into mammalian cells, there is evidence that translocations can be specifically induced in the presence of two TALEN pairs targeting different loci within the host genome $[59,60]$.

To circumvent these problems it could be beneficial to design viral vectors that contain both expression cassettes of a TALEN pair in one construct as conducted in the BV-based study [40]. This approach may decrease toxic side effects induced by the viral vector itself, because a decreased viral dose may be required for sufficient TALEN delivery. Depending on the number of RVD motifs of the respective TALEN, the size of a single TALEN expression cassette consisting of a promoter, the TALEN-gene and a polyadenylation signal can easily exceed 3.5 $\mathrm{kb}$. Keeping in mind that the specificity of TALENs correlates with the number of repeats, besides HCAdV and BV the majority of the commonly used vector systems (AdV, LV, AAV) fail in providing the appropriate capacity for two TALEN expression cassettes contained in one vector. However, internal ribosomal entry site (IRES)-based bicistronic expression cassettes or $2 \mathrm{~A}$ peptide linked multicistronic vectors may represent a potential solution. With respect to high expression levels of TALENs and duration of TALEN expression in transduced cells, expression of TALENs could be tightly controlled or only induced during a short period of time. Towards that end a possible solution would be the introduction of inducible promoter systems to control TALEN expression. Several inducible systems are well established as exemplified by the antibiotic-based TET-ON system [61] as well as mifepristone-inducible systems [62] to control protein expression. These machineries provide the opportunity to tightly control and monitor transgene expression. Especially antibioticinducible promoters could be of great importance for therapeutic

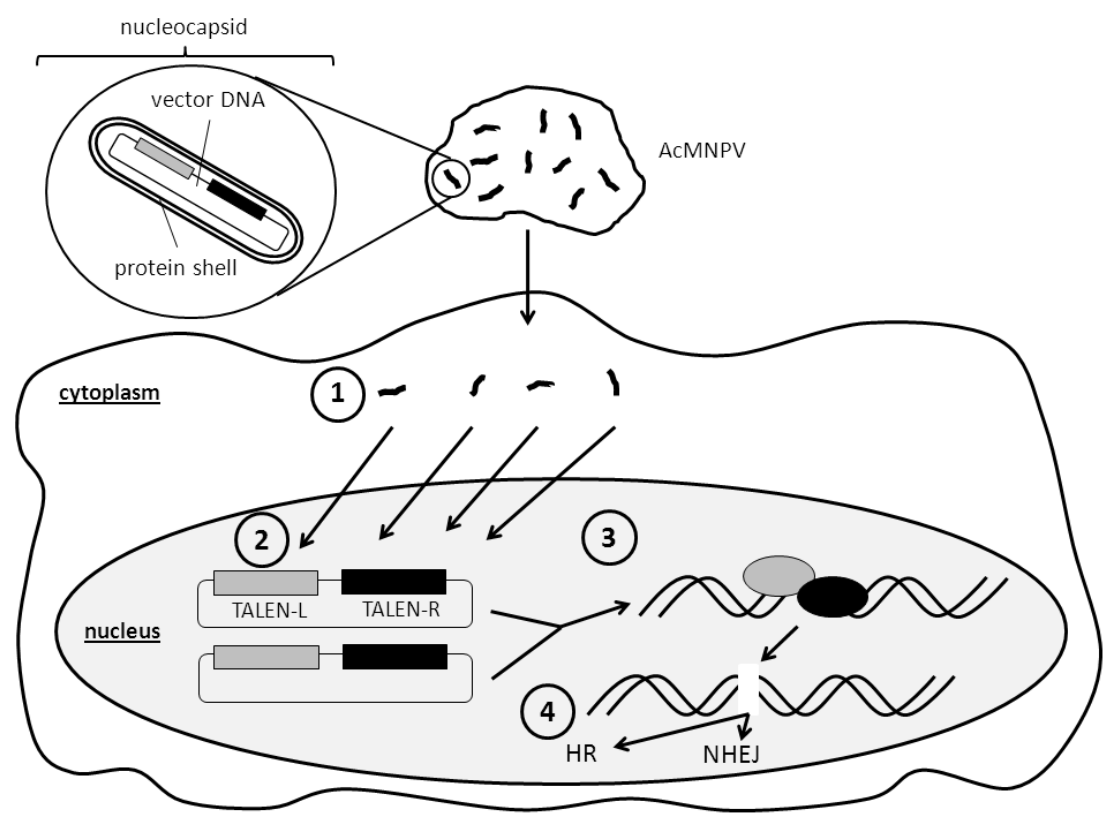

Figure 2: Schematic overview of TALEN expression via baculovirus derived vectors (AcMNPV). Virions of AcMNPVs contain a varying number of nucleocapsids each containing one vector genome carrying both expression cassettes of the TALEN pair. Transduction is mediated by membrane fusion of virion and host cell membranes and subsequent endosomal uptake (1). Next, release of nucleocapsids into the cytoplasm and subsequent transport of the genetic cargo on actin filaments to the nucleus occurs (2). After nuclear entry expression of the TALEN pair from circular double-stranded DNA vector genomes takes place which can then bind to the desired genomic target site (3). TALEN induced double-strand break then either leads to non-homologous end joining (NHEJ) or homologous recombination (HR) in the presence of respective donor DNA (4). AcMNPV: Autographa californica multiple nucleopolyhedrovirus; TALEN-L: left part of the TALEN pair; TALEN-R: right part of the TALEN pair; Grey boxes: TALEN-L expression cassette; Black boxes: TALEN-R expression cassette; Grey oval: TALEN-L protein; Black oval: TALEN-R protein. 
approaches because their inductors can be applied in a non-invasive manner.

\section{Future Viral Vectors for Delivery of TALENs}

Although LV vectors hold great promise for gene therapeutic applications, at the time they are not capable of sufficiently delivering functional TALEN pairs based on their current molecular design. However, in 2004 a novel version of a retroviral vector deficient for reverse transcriptase activity was designed, which was generated by deletions or mutations in the primer binding site necessary for the viral reverse transcription reaction [63]. Previous studies could show that these vectors provide highly efficient transduction rates in combination with transient transgene expression. Without the ability to reversely transcribe the viral RNA to DNA, the vector genome acts like mRNA molecules in the cytoplasm leading to short time expression of the desired transgene. With the possibility to express TALENs directly from RNA with a short life span, it may be possible to overcome the potential problem of toxicity and off-target effects. However, up to now only murine leukemia virus (MLV)-based retroviral systems have been invented to act only on the translational level, but in the future it may also be possible to design $\mathrm{LV}$ vectors which lack the function of reverse transcriptase. Most importantly, these new vectors would also circumvent the deletion of repetitive sequences within the TALE during the lentiviral reverse transcription reaction. It is of note, that LV vectors may also be able to provide required space for two TALEN encoding sequences contained in one vector. Depending on the number of repeats within each TALEN, it could be possible to translate both TALENs from one bicistronic mRNA template. To achieve that, both TALEN genes could be linked via an IRES sequence. Another strategy could be based on a multicistronic approach in which TALEN proteins could be linked via a $2 \mathrm{~A}$ peptide. However, at the time no data are available for these approaches but combining reverse transcriptase deficient $\mathrm{LV}$ vectors expressing a complete TALEN pair from one bicistronic RNA would offer a beneficial tool for further scientific and therapeutic studies.

So far the only platforms which can provide the necessary capacity for two complete TALEN expression cassette of large and specific TALENs are HCAdV and BV based vectors. Especially HCAdV vectors lacking all viral genes offer great potential for delivery of these large TALEN expression cassettes, because in contrast to BV vectors HCAdVs show only moderately induced immune responses in vivo. In combination with the possibility to strictly control duration of TALEN expression based on inducible promoters, the HCAdV system may hold great promise especially for sequence-specific genome engineering in vivo. BV based vectors instead provide a powerful tool for ex vivo approaches. For instance progenies of modified iPSCs using the BV vector system for TALEN delivery could play an emerging role in regenerative medicine.

Besides gene disruption due to TALEN activity in mammalian cells, another major goal of TALEN-based therapeutic approaches is the establishment of efficient strategies for gene correction. It could be shown that dsDNA breaks caused by TALENs induce homologous recombination in the presence of homologous sequences contained in the respective donor DNA [64-66]. This mechanism can be used to correct mutations associated with genetic diseases by transducing a DNA sequence homologous to the target sequence along with the TALEN encoding sequences. In the context of the ZFN technology, IDLVs [4] and first generation AdVs [67] were explored as donors providing DNA substrates for homologous recombination. However, efficiencies of homologous recombination for these systems are low and the molecular design especially of the donor DNA vectors needs to be improved.

Currently there are no data available for TALEN-based approaches in a clinical context. Due to the fact that this technology is relatively new only preclinical studies were performed until now. However, the ZFN technology could show some benefits for clinical applications $[22,68,69]$ and clinical trials were initiated using these nucleases to disrupt the human CCR5 locus in T cells in order to prevent entry of human immunodefiency virus 1 (HIV-1) into these cells (www.clinicaltrials. govNCT00842634, NCT01252641, NCT01044654). Keeping in mind that ZFN and TALE nucleases share the same mode of action, TALENs could be also be used in clinical applications in the future.

Notably, besides LV, AdV and BV vectors, also other viral vectors such as adeno-associated viral vectors (AAV vectors) for delivery of functional TALEN expression cassettes will be of interest in the future. However, it remains to be shown whether these approaches are feasible and whether they are more efficient compared to the currently available vector systems.

\section{Conflict of Interest}

Authors declare having no potential competing financial interests

\section{Acknowledgement}

This work was supported by the Persist project (EU Framework Programme 7) and the Else Kröner-Fresenius Foundation to A.E.

\section{References}

1. Cong L, Ran FA, Cox D, Lin S, Barretto R, et al. (2013) Multiplex genome engineering using CRISPR/Cas systems. Science 339: 819-823.

2. Mali P, Yang L, Esvelt KM, Aach J, Guell M, et al. (2013) RNA-guided human genome engineering via Cas9. Science 339: 823-826.

3. Bibikova M, Carroll D, Segal DJ, Trautman JK, Smith J, et al. (2001) Stimulation of homologous recombination through targeted cleavage by chimeric nucleases. Mol Cell Biol 21: 289-297.

4. Lombardo A, Genovese P, Beausejour CM, Colleoni S, Lee YL, et al. (2007) Gene editing in human stem cells using zinc finger nucleases and integrasedefective lentiviral vector delivery. Nat Biotechnol 25: 1298-1306.

5. Hockemeyer D, Soldner F, Beard C, Gao Q, Mitalipova M, et al. (2009) Efficient targeting of expressed and silent genes in human ESCs and iPSCs using zincfinger nucleases. Nat Biotechnol 27: 851-857.

6. Cermak T, Doyle EL, Christian M, Wang L, Zhang Y, et al. (2011) Efficient design and assembly of custom TALEN and other TAL effector-based constructs for DNA targeting. Nucleic Acids Res 39: e82.

7. Li T, Huang S, Zhao X, Wright DA, Carpenter S, et al. (2011) Modularly assembled designer TAL effector nucleases for targeted gene knockout and gene replacement in eukaryotes. Nucleic Acids Res 39: 6315-6325.

8. Boch J, Scholze H, Schornack S, Landgraf A, Hahn S, et al. (2009) Breaking the code of DNA binding specificity of TAL-type III effectors. Science 326: 15091512.

9. Neff KL, Argue DP, Ma AC, Lee HB, Clark KJ, et al. (2013) Mojo Hand, a TALEN design tool for genome editing applications. BMC Bioinformatics 14: 1.

10. Reyon D, Tsai SQ, Khayter C, Foden JA, Sander JD, et al. (2012) FLASH assembly of TALENs for high-throughput genome editing. Nat Biotechnol 30: $460-465$.

11. Schmid-Burgk JL, Schmidt T, Kaiser V, Höning K, Hornung V (2013) A ligationindependent cloning technique for high-throughput assembly of transcription activatorâ€"like effector genes. Nat Biotechnol 31: 76-81.

12. Uhde-Stone C, Gor N, Chin T, Huang J, Lu B (2013) A do-it-yourself protocol for simple transcription activator-like effector assembly. Biol Proced Online 15: 3.

13. Mussolino C, Morbitzer R, Lütge F, Dannemann N, Lahaye T, et al. (2011) A novel TALE nuclease scaffold enables high genome editing activity in combination with low toxicity. Nucleic Acids Res 39: 9283-9293. 
14. Hockemeyer D, Wang H, Kiani S, Lai CS, Gao Q, et al. (2011) Genetic engineering of human pluripotent cells using TALE nucleases. Nat Biotechno 29: $731-734$

15. Davies B, Davies G, Preece C, Puliyadi R, Szumska D, et al. (2013) Site specific mutation of the Zic2 locus by microinjection of TALEN mRNA in mouse CD1, C3H and C57BL/6J oocytes. PLoS One 8: e60216.

16. Sung YH, Baek IJ, Kim DH, Jeon J, Lee J, et al. (2013) Knockout mice created by TALEN-mediated gene targeting. Nat Biotechnol 31: 23-24.

17. Gaj T, Gersbach CA, Barbas CF 3rd (2013) ZFN, TALEN, and CRISPR/Casbased methods for genome engineering. Trends Biotechnol 31: 397-405.

18. Bloom K, Ely A, Mussolino C, Cathomen T, Arbuthnot $P$ (2013) Inactivation of hepatitis B virus replication in cultured cells and in vivo with engineered transcription activator-like effector nucleases. Mol Ther 21: 1889-1897.

19. Osborn MJ, Starker CG, McElroy AN, Webber BR, Riddle MJ, et al. (2013) TALEN-based gene correction for epidermolysis bullosa. Mol Ther 21: 11511159.

20. Ma N, Liao B, Zhang H, Wang L, Shan Y, et al. (2013) Transcription activatorlike effector nuclease (TALEN)-mediated gene correction in integration-free $\hat{~}^{2}$ thalassemia induced pluripotent stem cells. J Biol Chem 288: 34671-34679.

21. Li H, Haurigot $\mathrm{V}$, Doyon Y, Li T, Wong SY, et al. (2011) In vivo genome editing restores haemostasis in a mouse model of haemophilia. Nature 475: 217-221.

22. Perez EE, Wang J, Miller JC, Jouvenot Y, Kim KA, et al. (2008) Establishment of $\mathrm{HIV}-1$ resistance in CD4+ T cells by genome editing using zinc-finger nucleases. Nat Biotechnol 26: 808-816.

23. Andrews JL, Kadan MJ, Gorziglia MI, Kaleko M, Connelly S (2001) Generation and characterization of E1/E2a/E3/E4-deficient adenoviral vectors encoding human factor VIII. Mol Ther 3: 329-336.

24. Lusky M, Christ M, Rittner K, Dieterle A, Dreyer D, et al. (1998) In vitro and in vivo biology of recombinant adenovirus vectors with $\mathrm{E} 1, \mathrm{E} 1 / \mathrm{E} 2 \mathrm{~A}$, or E1/E4 deleted. J Virol 72: 2022-2032.

25. Yang Y, Ertl HC, Wilson JM (1994) MHC class I-restricted cytotoxic T lymphocytes to viral antigens destroy hepatocytes in mice infected with E1deleted recombinant adenoviruses. Immunity 1: 433-442.

26. Palmer D, Ng P (2003) Improved system for helper-dependent adenovira vector production. Mol Ther 8: 846-852.

27. Parks RJ, Chen L, Anton M, Sankar U, Rudnicki MA, et al. (1996) A helperdependent adenovirus vector system: removal of helper virus by Cre-mediated excision of the viral packaging signal. Proc Natl Acad Sci U S A 93: 1356513570.

28. Seiler MP, Cerullo V, Lee B (2007) Immune response to helper dependent adenoviral mediated liver gene therapy: challenges and prospects. Curr Gene Ther 7: 297-305

29. Jager L, Ehrhardt A (2009) Persistence of high-capacity adenoviral vectors as replication-defective monomeric genomes in vitro and in murine liver. Hum Gene Ther 20: 883-896.

30. Naldini L, Blömer U, Gallay P, Ory D, Mulligan R, et al. (1996) In vivo gene delivery and stable transduction of nondividing cells by a lentiviral vector Science 272: 263-267.

31. Zufferey R, Nagy D, Mandel RJ, Naldini L, Trono D (1997) Multiply attenuated lentiviral vector achieves efficient gene delivery in vivo. Nat Biotechnol 15: 871875 .

32. Madhan S, Prabakaran M, Kwang J (2010) Baculovirus as vaccine vectors. Curr Gene Ther 10: 201-213.

33. Schröder AR, Shinn P, Chen H, Berry C, Ecker JR, et al. (2002) HIV-1 integration in the human genome favors active genes and local hotspots. Cell 110: 521-529.

34. Mitchell RS, Beitzel BF, Schroder AR, Shinn P, Chen H, et al. (2004) Retrovira DNA integration: ASLV, HIV, and MLV show distinct target site preferences. PLoS Biol 2: E234.

35. Hacein-Bey-Abina S, Von Kalle C, Schmidt M, McCormack MP, Wulffraat N, et al. (2003) LMO2-associated clonal T cell proliferation in two patients after gene therapy for SCID-X1. Science 302: 415-419

36. Nightingale SJ, Hollis RP, Pepper KA, Petersen D, Yu XJ, et al. (2006) Transient gene expression by nonintegrating lentiviral vectors. Mol Ther 13: 1121-1132.

37. Mátrai J, Cantore A, Bartholomae CC, Annoni A, Wang W, et al. (2011) Hepatocyte-targeted expression by integrase-defective lentiviral vectors induces antigen-specific tolerance in mice with low genotoxic risk. Hepatology 53: 1696-1707.

38. Du J, Zeng J, Zhao Y, Boulaire J, Wang S (2010) The combined use of viral transcriptional and post-transcriptional regulatory elements to improve baculovirus-mediated transient gene expression in human embryonic stem cells. J Biosci Bioeng 109: 1-8.

39. Chen CY, Lin CY, Chen GY, Hu YC (2011) Baculovirus as a gene delivery vector: recent understandings of molecular alterations in transduced cells and latest applications. Biotechnol Adv 29: 618-631.

40. Zhu H, Lau CH, Goh SL, Liang Q, Chen C, et al. (2013) Baculoviral transduction facilitates TALEN-mediated targeted transgene integration and Cre/LoxP cassette exchange in human-induced pluripotent stem cells. Nucleic Acids Res 41: e180.

41. Jardin BA, Zhao Y, Selvaraj M, Montes J, Tran R, et al. (2008) Expression of SEAP (secreted alkaline phosphatase) by baculovirus mediated transduction of HEK 293 cells in a hollow fiber bioreactor system. J Biotechnol 135: 272-280.

42. Liu CY, Chen HZ, Chao YC (2010) Maximizing baculovirus-mediated foreign proteins expression in mammalian cells. Curr Gene Ther 10: 232-241.

43. Lucifora J, Durantel D, Belloni L, Barraud L, Villet S, et al. (2008) Initiation of hepatitis $B$ virus genome replication and production of infectious virus following delivery in HepG2 cells by novel recombinant baculovirus vector. J Gen Virol 89: 1819-1828.

44. Zheng H, Liu C, Zhuang J, Yuan S (2010) Baculovirus expression of cloned porcine arterivirus generates infectious particles in both insect and mammalian cells. J Biotechnol 150: 251-258.

45. Hu YC (2008) Baculoviral vectors for gene delivery: a review. Curr Gene Ther 8: $54-65$.

46. Hitchman RB, Possee RD, King LA (2009) Baculovirus expression systems for recombinant protein production in insect cells. Recent Pat Biotechnol 3: 46-54

47. Wang S, Balasundaram G (2010) Potential cancer gene therapy by baculovira transduction. Curr Gene Ther 10: 214-225.

48. Hervas-Stubbs S, Rueda P, Lopez L, Leclerc C (2007) Insect baculoviruses strongly potentiate adaptive immune responses by inducing type I IFN. J Immunol 178: 2361-2369.

49. Kitajima M, Abe T, Miyano-Kurosaki N, Taniguchi M, Nakayama T, et al. (2008) Induction of natural killer cell-dependent antitumor immunity by the Autographa californica multiple nuclear polyhedrosis virus. Mol Ther 16: 261-268.

50. Ge J, Huang Y, Hu X, Zhong J (2007) A surface-modified baculovirus vector with improved gene delivery to B-lymphocytic cells. J Biotechnol 129: 367-372.

51. Kaname Y, Tani H, Kataoka C, Shiokawa M, Taguwa S, et al. (2010) Acquisition of complement resistance through incorporation of CD55/decay-accelerating factor into viral particles bearing baculovirus GP64. J Virol 84: 3210-3219.

52. Holkers M, de Vries AA, Gonçalves MA (2012) Nonspaced inverted DNA repeats are preferential targets for homology-directed gene repair in mammalian cells. Nucleic Acids Res 40: 1984-1999.

53. Kotin RM, Menninger JC, Ward DC, Berns KI (1991) Mapping and direct visualization of a region-specific viral DNA integration site on chromosome 19q13-qter. Genomics 10: 831-834

54. Pathak VK, Temin HM (1990) Broad spectrum of in vivo forward mutations hypermutations, and mutational hotspots in a retroviral shuttle vector after single replication cycle: deletions and deletions with insertions. Proc Natl Acad Sci U S A 87: 6024-6028.

55. Julias JG, Hash D, Pathak VK (1995) E- vectors: development of novel selfinactivating and self-activating retroviral vectors for safer gene therapy. J Viro 69: 6839-6846.

56. Delviks KA, Pathak VK (1999) Effect of distance between homologous sequences and 3 ' homology on the frequency of retroviral reverse transcriptase template switching. J Virol 73: 7923-7932.

57. Rhode BW, Emerman M, Temin HM (1987) Instability of large direct repeats in retrovirus vectors. J Virol 61: 925-927. 
Citation: Bergmann T, Schulz E, Ehrhardt A (2014) Progress and Problems with Viral Vectors for Delivery of Talens. J Mol Genet Med 7: 096 doi: 10.4172/1747-0862.1000096

Page 7 of 7

58. Holkers M, Maggio I, Liu J, Janssen JM, Miselli F, et al. (2013) Differential integrity of TALE nuclease genes following adenoviral and lentiviral vector gene transfer into human cells. Nucleic Acids Res 41: e63.

59. Xiao A, Wang Z, Hu Y, Wu Y, Luo Z, et al. (2013) Chromosomal deletions and inversions mediated by TALENs and CRISPR/Cas in zebrafish. Nucleic Acids Res 41: e141.

60. Piganeau M, Ghezraoui H, De Cian A, Guittat L, Tomishima M, et al. (2013) Cancer translocations in human cells induced by zinc finger and TALE nucleases. Genome Res 23: 1182-1193.

61. Gossen M, Bujard H (1992) Tight control of gene expression in mammalian cells by tetracycline-responsive promoters. Proc Natl Acad Sci U S A 89: 5547 5551.

62. Wang Y, O'Malley BW Jr, Tsai SY, O'Malley BW (1994) A regulatory system for use in gene transfer. Proc Natl Acad Sci U S A 91: 8180-8184.

63. Galla M, Will E, Kraunus J, Chen L, Baum C (2004)Retroviral pseudotransduction for targeted cell manipulation. Mol Cell 16: 309-315.

64. Zu Y, Tong X, Wang Z, Liu D, Pan R, et al. (2013) TALEN-mediated precise genome modification by homologous recombination in zebrafish. Nat Methods 10: $329-331$.

65. Bedell VM, Wang Y, Campbell JM, Poshusta TL, Starker CG, et al. (2012) In vivo genome editing using a high-efficiency TALEN system. Nature 491: 114118.

66. Ousterout DG, Perez-Pinera P, Thakore PI, Kabadi AM, Brown MT, et al. (2013) Reading frame correction by targeted genome editing restores dystrophin expression in cells from duchenne muscular dystrophy patients. Mol Ther 21 1718-1726.

67. Coluccio A, Miselli F, Lombardo A, Marconi A, Malagoli Tagliazucchi G, et al (2013) Targeted gene addition in human epithelial stem cells by zinc-finger nuclease-mediated homologous recombination. Mol Ther 21: 1695-1704.

68. Holt N, Wang J, Kim K, Friedman G, Wang X, et al. (2010) Human hematopoietic stem/progenitor cells modified by zinc-finger nucleases targeted to CCR5 control HIV-1 in vivo. Nat Biotechnol 28: 839-847.

69. Voit RA, McMahon MA, Sawyer SL, Porteus MH (2013) Generation of an HIV resistant T-cell line by targeted "stacking" of restriction factors. Mol Ther 21 786-795. 